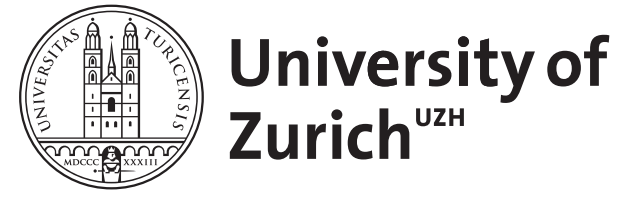

Zurich Open Repository and Archive

University of Zurich

University Library

Strickhofstrasse 39

CH-8057 Zurich

www.zora.uzh.ch

Year: 2007

\title{
Akute gastrointestinale Infektionen
}

Fried, M

DOI: https://doi.org/10.1007/s11377-007-0086-5

Posted at the Zurich Open Repository and Archive, University of Zurich

ZORA URL: https://doi.org/10.5167/uzh-155757

Journal Article

Published Version

Originally published at:

Fried, M (2007). Akute gastrointestinale Infektionen. Der Gastroenterologe, 2(3):159-160.

DOI: https://doi.org/10.1007/s11377-007-0086-5 
Gastroenterologe $2007 \cdot 2: 159-160$ DOI 10.1007/s11377-007-0086-5

Online publiziert: 11. April 2007

๑) Springer Medizin Verlag 2007

M. Fried

Klinik für Gastroenterologie und Hepatologie, Universitätsspital Zürich

\section{Akute gastrointestinale Infektionen}

Akute Infektionen des Gastrointestinaltraktes sind häufige Probleme in der Praxis. Ihr klinisches Erscheinungsbild reicht von der asymptomatischen Infektion bis zur schweren Systemerkrankung, die zum Tod des Patienten führen kann. Ziel dieser Folge von Artikeln über akute Infektionen im Bereich des Darmes und der Leber ist es, dem Kliniker eine Zusammenfassung der wichtigen Fakten über diese verbreiteten akuten Infektionserkrankungen mit einer Betonung der modernen diagnostischen und therapeutischen Aspekte zur Verfügung zu stellen.

Infektiöse Diarrhöerkrankungen sind bei Reisen häufige Manifestationen akuter Infektionen, insbesondere mit enterotoxigenen E. coli, Salmonellen, Shigellen und Campylobacter. Wie Pitzurra et al. in ihrem Beitrag aufzeigen, spielen verschiedene Faktoren bei der Entstehung der Reisediarrhö eine besondere Rolle. So ist die Inzidenzrate - entgegen landläufiger Meinung - bei den Hotels der Luxusklasse eher höher als in den einfacheren Hotelkategorien. Erstaunlich ist, dass sich nur ein sehr geringer Teil der Reisenden an die bekannten Regeln zur Vermeidung einer durch Nahrungsmittel übertragene Infektionen halten. Auffallend ist die Häufung von Norovirus-Epidemien auf Kreuzfahrten. Wichtige Fortschritte in der Prophylaxe sind bei der Entwicklung von Impfstoffen zu verzeichnen. So sind Vakzine gegen Choleravibrionen/E.-coli-Enterotoxin, Rotaviren und bald auch Shigellen und Campyloacter verfügbar, die vor allem zur Prophylaxe bei Risikogruppen sinnvoll sind. Bei den meisten Patienten ist die Reisediarrhö ein zeitlich umschriebenes Ereignis, jedoch kann es bei einer Untergruppe zu langfristigen Beschwerden (Diarrhö, Abdominalschmerzen) kommen, auch wenn die Infektion längst eliminiert ist. Dabei handelt es sich um ein sogenanntes postinfektiöses Reizdarmsyndrom, das eine entsprechende Behandlung erfordert.

\section{( ) Grundsätzlich können alle Antibiotika eine C.-difficile- Infektion auslösen}

Eine immer größere Rolle spielt die Clostridium-difficile-assoziierte Diarrhö, insbesondere nach Antibiotikabehandlungen und häufig bei Patienten im Spital. Rampini et al. zeigen in ihrem Übersichtsartikel, dass diese Infektion keineswegs immer harmlos verläuft, sie kann mit einer relativ hohen Mortalität und beträchtlichen Kosten vergesellschaftet sein, was insbesondere bei einem neuen hochvirulenten Stamm beobachtet wurde. Neben den "klassischen" auslösenden Antibiotika Ampicillin, Cephalosporine und Clindamycin können grundsätzlich alle Antibiotika, wenn auch mit unterschiedlichem Potenzial, zu einer Clostridium-difficile-Infektion führen. Pathogenetisch interessant und Anlass für aktuelle Studien ist die kürzlich beobachtete erhöhte Prävalenz einer C.-difficile-Infektion bei Patienten mit M. Crohn oder Colitis ulcerosa. Therapeutisch ist nach wie vor Metronidazol Mittel der ersten Wahl, gefolgt von Vancomycin, das peroral verabreicht werden muss. Dieses teure Medikament hat auch den Nachteil der möglichen Selektion vancomycinresistenter Enterokokken. Häufig wird die hohe Rezidivrate von etwa $20 \%$ dieser Infektion übersehen, was eine erneute Behandlung erfordert. Die Beachtung der Hygiene im Spital, insbesondere die regelmäßige Händedesinfektion mit Alkohol sowie die Händewaschung mit Seife, sind nach wie vor die besten und einfachsten Maßnahmen zur Ver- 
meidung dieser und anderer nosokomialer Infektionen.

Rotaviren bei Säuglingen und Kleinkindern und Noroviren in allen Altersgruppen sind zunehmend häufige Ursachen einer akuten Gastroenteritis und können für Epidemien, z. B. in Alters- und Pflegeheimen, verantwortlich sein. W. Zingg zeigt in seinem Beitrag, dass die zunehmend hohe Zahl von Norovirus-Epidemien durch die hohe Infektiosität und genetische Variabilität dieses Virus erklärt werden kann. Hygienische Aspekte, insbesondere Isolation der betroffenen Patienten und Desinfektionsmaßnahmen, spielen bei ihrer Bekämpfung eine besondere Rolle. In den USA ist seit kurzem eine Rotavirus-Vakzine zugelassen worden, eine Impfung gegen das Norovirus steht leider noch nicht zur Verfügung. Therapeutisch sollte möglichst eine orale Rehydratation durchgeführt werden, sie ist einfacher, billiger und führt seltener als die Infusionstherapie zu Komplikationen.

(? Norovirus-Epidemien sind durch eine hohe Infektiosität sowie genetische Variabiltiät des Virus zu erklären

Neben den akuten Diarrhöerkrankungen gehören die akuten Virushepatitiden, insbesondere die Hepatitis $A$, zu den häufigsten akuten Infektionskrankheiten. Die Palette der klinischen Manifestationen reicht von einem leichten grippalen Infekt bis zur fulminanten Hepatitis, die zum Tod des Patienten führen kann. T. Gerlach und B. Müllhaupt führen in ihrem Übersichtsbeitrag aus, dass die akute Virushepatitis sich häufig nur durch unspezifische Symptome, wie Müdigkeit, und Myalgien äußert, aber auch asymptomatisch verlaufen kann und nur bei einem Teil der Patienten einen Ikterus verursacht. Dies erklärt auch, dass die akute Virushepatitis häufig nicht erkannt wird. In den meisten Fällen erfordert die akute Hepatitis-A- und auch-B-Infektion keine Therapie, bei der seltenen fulminanten Verlaufsform der Hepatitis B ist jedoch eine Behandlung in einem hepatologischen Zentrum erforderlich, wobei auch die Option einer Lebertransplantation geprüft werden muss. Bei der akuten Hepatitis C ist ei$n e$ rasche Behandlung mit Interferon- $\alpha$ oder pegyliertem Interferon- $\alpha$ indiziert.
In diesem Band haben auf ihrem Gebiet namhafte Experten in didaktisch ansprechender Weise neueste Erkenntnisse über die Diagnose, Pathophysiologie und Therapie der akuten Infektionen im Gastrointestinaltrakt zusammengestellt und konzis präsentiert. Dieser Band ist gleichermaßen für den Arzt in der Praxis wie für den im Spital eine wichtige Informationsquelle für den klinischen Alltag.

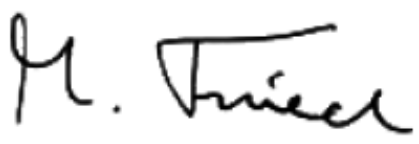

M. Fried, Zürich

\section{Korrespondierender Autor Prof. Dr. M. Fried}

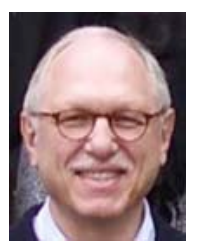

Klinik für Gastroenterologie und Hepatologie, Universitätsspital Zürich, Rämistraße 100, 8091 Zürich Michael.Fried@usz.ch
„Der Gastroenterologe“ bietet Ihnen jeden Monat umfassende und aktuelle Beiträge zu interessanten Themenschwerpunkten aus allen Bereichen der Gastroenterologie und Hepatologie.

\section{7 (Änderungen vorbehalten)}

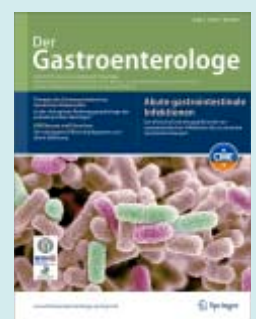

$$
\begin{array}{ll}
\text { - Heft 1/07 } & \text { Leberzellkarzinom } \\
\text { - Heft 2/07 } & \begin{array}{l}
\text { Neue Techniken } \\
\text { in der Endoskopie }
\end{array} \\
\text { - Heft 3/07 } & \begin{array}{l}
\text { Neue gastrointestinale } \\
\text { Infektionen }
\end{array} \\
& \text { Leberzirrhose und ihre } \\
\text { - Heft 4/07 } & \text { Komplikationen } \\
\text { - Heft 5/07 } & \text { Früherkennung } \\
& \text { und Prävention maligner } \\
& \text { Erkrankungen im Gl-Trakt }
\end{array}
$$

- Heft 6/07 CED

\section{www.DerGastroentero- loge.springer.de}

Haben Sie noch Fragen oder Anmerkungen? Wir freuen uns über Ihre E-mail an: birgit.wasser@springer.com

Ihre Redaktion

Fachzeitschriften Medizin/Psychologie 